\title{
Reciprocal space mapping and strain scanning using X-ray diffraction microscopy
}

Poulsen, H. F.; Cook, P. K.; Leemreize, H.; Pedersen, A. F.; Yildirim, C.; Kutsal, M.; Jakobsen, A. C.; Trujillo, J. X.; Ormstrup, J.; Detlefs, C.

Published in:

Journal of Applied Crystallography

Link to article, DOI:

$10.1107 / \mathrm{S} 1600576718011378$

Publication date:

2018

Document Version

Publisher's PDF, also known as Version of record

Link back to DTU Orbit

Citation (APA):

Poulsen, H. F., Cook, P. K., Leemreize, H., Pedersen, A. F., Yildirim, C., Kutsal, M., Jakobsen, A. C., Trujillo, J. X., Ormstrup, J., \& Detlefs, C. (2018). Reciprocal space mapping and strain scanning using X-ray diffraction microscopy. Journal of Applied Crystallography, 51(5). https://doi.org/10.1107/S1600576718011378

\section{General rights}

Copyright and moral rights for the publications made accessible in the public portal are retained by the authors and/or other copyright owners and it is a condition of accessing publications that users recognise and abide by the legal requirements associated with these rights.

- Users may download and print one copy of any publication from the public portal for the purpose of private study or research.

- You may not further distribute the material or use it for any profit-making activity or commercial gain

- You may freely distribute the URL identifying the publication in the public portal 


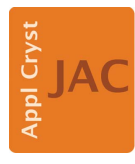

JOURNAL OF APPLIED CRYSTALLOGRAPHY

ISSN 1600-5767

Received 18 June 2018

Accepted 9 August 2018

Edited by A. Borbély, Ecole National Supérieure des Mines, Saint-Etienne, France

Keywords: X-ray diffraction microscopy; diffraction contrast tomography; structural characterization; synchrotron radiation; tomography; diffraction imaging.

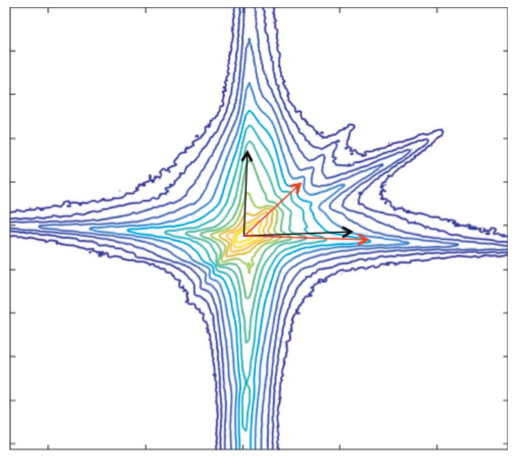

C 2018 International Union of Crystallography

\section{Reciprocal space mapping and strain scanning using $\mathrm{X}$-ray diffraction microscopy}

\author{
H. F. Poulsen, ${ }^{a *}$ P. K. Cook, ${ }^{b}$ H. Leemreize, ${ }^{c, a}$ A. F. Pedersen, ${ }^{a}$ C. Yildirim, ${ }^{b, d}$ \\ M. Kutsal, ${ }^{b}$ A. C. Jakobsen, ${ }^{a}$ J. X. Trujillo, ${ }^{e}$ J. Ormstrup ${ }^{a}$ and C. Detlefs ${ }^{b}$ \\ ${ }^{\mathbf{a}}$ Department of Physics, Technical University of Denmark, 2800 Kongens Lyngby, Denmark, ${ }^{\mathbf{b}}$ European Synchrotron \\ Radiation Facility, 71 avenue des Martyrs, CS40220, 38043 Grenoble Cedex 9, France, ${ }^{\mathbf{c} D a n i s h}$ Technological Institute, \\ Denmark, dOCAS, J. F. Kennedylaan 3, 9060 Zelzate, Belgium, and ${ }^{\mathbf{e}}$ Department of Energy Storage and Conversion, \\ Technical University of Denmark, 4000 Roskilde, Denmark. *Correspondence e-mail: hfpo@fysik.dtu.dk
}

Dark-field X-ray microscopy is a new full-field imaging technique for nondestructively mapping the structure of deeply embedded crystalline elements in three dimensions. Placing an objective in the diffracted beam generates a magnified projection image of a local volume. By placing a detector in the back focal plane, high-resolution reciprocal space maps are generated for the local volume. Geometrical optics is used to provide analytical expressions for the resolution and range of the reciprocal space maps and the associated field of view in the sample plane. To understand the effects of coherence a comparison is made with wavefront simulations using the fractional Fourier transform. Reciprocal space mapping is demonstrated experimentally at an $\mathrm{X}$-ray energy of $15.6 \mathrm{keV}$. The resolution function exhibits suppressed streaks and an FWHM resolution in all directions of $\Delta Q / Q=4 \times 10^{-5}$ or better. It is demonstrated by simulations that scanning a square aperture in the back focal plane enables strain mapping with no loss in resolution to be combined with a spatial resolution of $100 \mathrm{~nm}$.

\section{Introduction}

Dark-field X-ray microscopy (DFXRM) is a new full-field imaging technique for mapping crystallographic features in bulk specimens in three dimensions (Simons et al., 2015). Similar to bright-field X-ray microscopy (Schroer et al., 2001), an objective lens is inserted between the sample and a highresolution two-dimensional detector, but in this case in the Bragg diffracted beam. This enables nondestructive mapping of structure, orientation and strain within deeply embedded crystalline elements (Simons et al., 2015). A first implementation at beamline ID06 at the European Synchrotron (ESRF) is based on the use of a monochromatic beam in the 15-35 keV range and a compound refractive lens (CRL) (Snigirev et al., 1996) as objective. The magnification and field of view can be modified by changing the focal length of this objective. Lens imperfections currently limit the spatial resolution to $\sim 100 \mathrm{~nm}$.

In combination with coarse-scale grain mapping methods such as three-dimensional X-ray diffraction (Poulsen, 2004, 2012; Hefferan et al., 2012) and diffraction contrast tomography (King et al., 2008; Ludwig et al., 2009), DFXRM has proven to be a powerful method for multi-scale studies of polycrystals and their dynamics (Simons et al., 2016). First applications include work on the processing of plastically deformed metals (Ahl et al., 2015), the distribution of strain and orientation gradients in ferroelectrics (Simons et al., 2018), 
and the three-dimensional mapping of dislocations (Simons et al., 2016), as well as studies of biominerals (Cook, 2018).

Poulsen et al. (2017) provided a detailed description of the optics of the image plane of this microscope, including parameters such as numerical aperture, vignetting, and the resolution in both direct and reciprocal space. It was shown that the resolution function in reciprocal space can be highly anisotropic and can vary as a function of position within the field of view. Procedures for sampling and conservation of integrated intensities were presented. We shall refer to this work as Paper 1 throughout.

Similar to classical light microscopy and transmission electron microscopy (Williams \& Carter, 2009), the hard X-ray microscope is associated with a back focal plane (BFP). The intensity distribution in the BFP is equivalent to the distribution in the Fraunhofer far-field limit. This has been utilized for bright-field microscopy studies (Bosak et al., 2010; Ershov et al., 2013; Falch et al., 2018).

Complementary to Paper 1 , in this work we provide a detailed description of the optics of the BFP in the dark-field mode. Analytical expressions are derived from a thick-lens ray-transfer-matrix formalism following Simons et al. (2017). When relevant, this work is supplemented by full-scale wavefront simulations based on fractional Fourier transforms (Ozaktas \& Mendlovic, 1995; Le Bolloch et al., 2012; Pedersen et al., 2018). Procedures for reciprocal space mapping based on placing a two-dimensional detector in the BFP are presented. Next, we consider placing an aperture in the BFP in combination with a detector in the imaging plane. It is shown that high strain resolution may be obtained without losing spatial resolution. In $\S 4$, the reciprocal space mapping is demonstrated by an experimental study at $15.6 \mathrm{keV}$.

\section{Geometry and formalism}

\subsection{Dark-field microscopy geometry}

The geometry of the dark-field X-ray microscope is illustrated in Fig. 1. The sample goniometer provides a base tilt, $\mu$, a rotation, $\omega$, and two orthogonal sample tilts, $\chi$ and $\phi$. The incident beam is defined by a slit close to the source and/or by a condenser. It is characterized by angular divergences $\Delta \zeta_{\mathrm{v}}$ and $\Delta \zeta_{\mathrm{h}}$ in the vertical and horizontal directions, respectively, and by an energy bandwidth $\Delta E / E$. The motors $\chi, \phi$ and $\mu$ are used to orient an embedded crystalline element of choice (e.g. a grain or domain) such that it is in the Laue condition with its diffraction vector, $\mathbf{Q}$, parallel to the rotation axis $\omega$, implying that $\mathbf{Q}$ remains in the diffraction condition at all values of $\omega$ (the so-called topo-tomography setting). The direction of the optical axis of the diffracted beam is described by the scattering angle, $2 \theta$, and the azimuthal angle, $\eta$ (Fig. 1).

The objective is in the following a CRL comprising $N$ identical parabolic shaped lenslets with a radius of curvature $R$ and a distance between the centers of adjacent lenslets $T$. Let the linear attenuation coefficient and the refractive index decrement of the lens material be $\mu_{\text {att }}$ and $\delta$, respectively. The objective magnifies the diffracted beam by a factor $\mathcal{M}_{\mathrm{CRL}}$ and generates an inverted two-dimensional image in the image plane. The distance from the sample plane to the front of the objective is $d_{1}$, and the distance between sample plane and image plane is $d_{1}+N T+d_{2}$ (see Fig. 1). With $N T$ typically of the same order as $d_{1}$, a thick-lens description is required. Simons et al. (2017) provided a comprehensive thick-lens description for the bright-field case using a ray-transfer-matrix (RTM) approach. In Paper 1, the same RTM formalism is used to provide equations for the dark-field case in relation to direct and reciprocal space resolution functions and a discussion of sampling strategies.

Three equations from Paper 1 of key interest for this work are

$$
\begin{gathered}
f_{N}=f \varphi \cot (\varphi N), \\
\sigma_{\mathrm{a}} \simeq \delta \frac{\mathcal{M}_{\mathrm{CRL}}}{\mathcal{M}_{\mathrm{CRL}}+1}\left(\frac{2 N}{\mu_{\mathrm{att}} R}\right)^{1 / 2}, \\
\sigma_{\mathrm{v}}=\frac{2 \delta}{\mu_{\mathrm{att}} \sigma_{\mathrm{a}}}\left[N^{2} \varphi^{2}-\sin ^{2}(N \varphi)\right]^{1 / 2} .
\end{gathered}
$$

Here $f=R /(2 \delta)$ is the focal length of one lenslet, $\varphi=(T / f)^{1 / 2}$, and $f_{N}$ is the focal length of the CRL and therefore the distance from the end of the objective to the back focal plane (cf. Fig. 1). $\sigma_{\mathrm{a}}$ is the r.m.s. width of the angular attenuation profile, describing the numerical aperture. $\sigma_{\mathrm{v}}$ is the r.m.s. width of the Gaussian distribution associated with vignetting.

Following Paper 1, for simplicity in the following we assume $\omega=\eta=0$. Furthermore we introduce two direct space coordinate systems: the imaging system - defined by $\hat{x}$ being parallel to the diffracted beam and $\hat{y}$ perpendicular to the incoming beam in the horizontal plane - and a reference system offset from the imaging system by an angle $\theta$ around the common $y$ axis. In both cases we can define colinear reciprocal space coordinate systems. For the reference system the coordinates are $\left(\hat{q}_{\text {rock }}, \hat{q}_{\text {roll }}, \hat{q}_{\|}\right)$. Here 'rock' refers to the

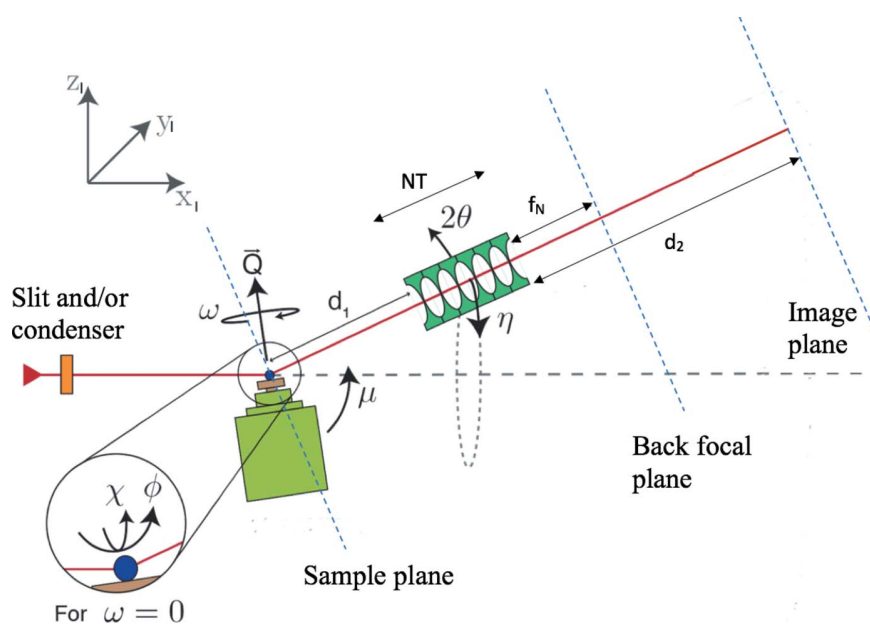

Figure 1

Principle of dark-field X-ray microscopy. The red line between the pivotal point of the goniometer and the detector (image plane) is the optical axis of the diffracted beam. The laboratory coordinate system $\left(x_{1}, y_{1}, z_{1}\right)$ is shown. See also main text. 
transverse in-plane 'rocking' direction of classical two-axis diffractometers, 'roll' to the out-of-plane 'rolling' around the incoming beam and $\|$ to the longitudinal (radial) direction in reciprocal space $\left(\hat{q}_{\|}\right.$is parallel to $\left.\mathbf{Q}\right)$. The corresponding coordinates for reciprocal space associated with the imaging system are defined as $\left(\hat{q}_{\text {rock' }}, \hat{q}_{\text {roll }}, \hat{q}_{2 \theta}\right)$. For more general settings and details of coordinate transforms see Paper 1.

\subsection{Imaging in the back focal plane}

In an ideal imaging system with a fully coherent beam, the (amplitude, phase) field in the BFP is a Fourier transform of the (amplitude, phase) field in the sample plane (Goodman, 2005). The BFP is in our case a plane perpendicular to the optical axis and is located at a distance of one focal length from the exit of the objective, as given by equation (1). Let $\left(\hat{y}_{\mathrm{B}}, \hat{z}_{\mathrm{B}}\right)$ span the BFP and be colinear with the axes $\left(\hat{y}_{\mathrm{s}}, \hat{z}_{\mathrm{s}}\right)$ in the sample plane. Then in geometrical optics there is a one-toone correspondence between coordinates $\left(y_{\mathrm{B}}, z_{\mathrm{B}}\right)$ and angles $\left(\xi_{y s}, \xi_{z \mathrm{~s}}\right)$ with respect to the optical axis - as measured in the sample plane. Using the RTM formalism, we have ( $c f$. Simons et al., 2017)

$$
\begin{aligned}
& y_{\mathrm{B}}=\frac{f_{N}}{\cos (N \varphi)} \xi_{y \mathrm{~s}}, \\
& z_{\mathrm{B}}=\frac{f_{N}}{\cos (N \varphi)} \xi_{z \mathrm{~s}} .
\end{aligned}
$$

These expressions are independent of the position in the sample space.

\section{Reciprocal space mapping}

In a scattering geometry where the optical axis of the objective is aligned with the center of a Bragg diffracted beam, the angular deviations $\xi_{y \mathrm{~s}}$ and $\xi_{z \mathrm{~s}}$ are related to reciprocal space. Let $\left(\Delta Q_{\text {rock' }^{\prime}} /\left|\mathbf{Q}_{0}\right|, \Delta Q_{\text {roll }} /\left|\mathbf{Q}_{0}\right|, \Delta Q_{2 \theta} /\left|\mathbf{Q}_{0}\right|\right)$ be the deviation from the nominal Bragg lattice point, $\mathbf{Q}_{0}$. Then, following equations (52) and (71) in Paper 1, we have

$$
\begin{gathered}
y_{\mathrm{B}}=\frac{2 \sin (\theta) f_{N}}{\cos (N \varphi)} \frac{\Delta Q_{\text {roll }}}{\left|\mathbf{Q}_{0}\right|}, \\
z_{\mathrm{B}}=\frac{2 \sin (\theta) f_{N}}{\cos (N \varphi)} \frac{\Delta Q_{2 \theta}}{\left|\mathbf{Q}_{0}\right|} .
\end{gathered}
$$

The third direction in reciprocal space - when described by the imaging system coordinates - is $\hat{q}_{\text {rock' }}$. However, the parameter that can easily be varied experimentally is $\hat{q}_{\text {rock }}$, as this is defined by the 'rocking angle', the position of either $\mu$ or $\phi$ (cf. Fig. 1). Hence, the experimental data, as defined by detector coordinates and rocking angle, are not in an orthogonal system.

To generate data in an orthogonal system one therefore needs to interpolate. In the imaging coordinate system this implies the following relationship:

$$
\frac{\Delta Q_{\text {rock' }^{\prime}}}{\left|\mathbf{Q}_{0}\right|}=\left(\phi-\phi_{0}\right) \cos (\theta),
$$

$$
\begin{gathered}
\frac{\Delta Q_{\text {roll }}}{\left|\mathbf{Q}_{0}\right|}=\frac{\cos (N \varphi)}{2 \sin (\theta) f_{N}} y_{\mathrm{B}}, \\
\frac{\Delta Q_{2 \theta}}{\left|\mathbf{Q}_{0}\right|}=\frac{\cos (N \varphi)}{2 \sin (\theta) f_{N}} z_{\mathrm{B}}-\left(\phi-\phi_{0}\right) \sin (\theta) .
\end{gathered}
$$

Here $\phi_{0}$ corresponds to the maximum of the rocking curve, and to $\mu$ being equal to the nominal Bragg angle.

\subsection{The effect of attenuation}

It is relevant to determine the range of the reciprocal space map and to know which area in the sample plane the reciprocal space map refers to. Both properties are defined by the attenuation of the CRL. Analytical expressions for the imaging plane are provided in Paper 1, which with simple modifications can be adapted to the BFP geometry.

For the point in the sample plane that is on the optical axis, the attenuation gives rise to an angular acceptance which is a Gaussian distribution defined by the numerical aperture. The r.m.s. width of the range in normalized reciprocal space $\mathbf{q}=\mathbf{Q} /\left|\mathbf{Q}_{0}\right|$ becomes

$$
\sigma_{q}=\frac{\sigma_{\mathrm{a}}}{2 \sin (\theta)} .
$$

For the point in reciprocal space which corresponds to the center of the region mapped, the intensity contributions from points in the sample plane are weighted with a Gaussian attenuation function, the vignetting. The width of this defines the field of view: the r.m.s. value is $\sigma_{\mathrm{v}}$, as expressed by equation (3).

The attenuation for the general off-axis case is given by equation (24) of Simons et al. (2017). Consider a ray emerging from a point in the sample plane with position $\mathbf{r}_{\mathrm{s}}$ and at a distance $\mathbf{q}_{r}$ to the nominal center in the (normalized) $\left(q_{\text {roll }}, q_{2 \theta}\right)$ plane. The attenuation then becomes a product of three terms:

$$
\begin{aligned}
& \operatorname{Att}\left(r_{\mathrm{s}}, \xi_{\mathrm{s}}\right)=\exp \left(-\mu N T_{\mathrm{w}}\right) \Omega\left(\mathbf{r}_{\mathrm{s}}, \mathbf{q}_{\mathrm{r}}\right) \operatorname{Vign}\left(\mathbf{r}_{\mathrm{s}}\right), \\
& =\exp \left(-\mu N T_{\mathrm{w}}\right) \exp \left(\frac{-\left\{q_{r}+[\gamma / 2 \sin (\theta)] r_{\mathrm{s}}\right\}^{2}}{2 \sigma_{q}^{2}}\right) \\
& \quad \times \exp \left(-\frac{r_{\mathrm{s}}^{2}}{2 \sigma_{\mathrm{v}}^{2}}\right) .
\end{aligned}
$$

The first term reflects the attenuation caused by the web distances, $T_{\mathrm{w}}$, between neighboring apices in the CRL. The second describes the angular acceptance, while the third is the vignetting term.

It appears that the vignetting in the sample plane remains the same as in the on-axis case. The middle term indicates that the range in reciprocal space is also unaltered, but the center position in reciprocal space shifts with varying $r_{\mathrm{s}}$ by $\gamma r_{\mathrm{s}} / 2 \sin (\theta)$. Expressions for $\gamma$ are provided in the supplementary materials of Simons et al. (2017). For long to medium focal distances we have to a good approximation

$$
1 / \gamma=\left(d_{1}^{2}+f^{2} \varphi^{2}\right)^{1 / 2}
$$

Typical numerical values for $1 / \gamma$ are $10-30 \mathrm{~cm}$. 


\subsection{Reciprocal space resolution}

Initially we will be concerned with the widths - in three orthogonal directions - of the reciprocal space resolution function and shall neglect low-intensity tails. We anticipate that these widths are governed by the divergence and the energy bandwidth of the incoming beam. Furthermore we shall assume the incoming beam to be Gaussian. Such a model was derived for the reciprocal space resolution function in the image plane in $\$ 4.1$ of Paper 1 . Applying a similar approach here for the BFP, we treat the divergence as small perturbations $\left(\zeta_{\mathrm{h}, \mathrm{v}}\right)$ to the horizontal and vertical components of the incident beam vector, $\mathbf{k}_{\text {in }}$, and the energy bandwidth in the form of a longitudinal perturbation, $\varepsilon=\Delta E / E=\Delta k / k$, to both incident and diffracted beam vectors. In the imaging coordinate system the deviations from the nominal incident and diffracted wavevectors are thus

$$
\begin{gathered}
\Delta \mathbf{k}_{\text {in }}=k\left[\begin{array}{ccc}
\cos (2 \theta) & 0 & \sin (2 \theta) \\
0 & 1 & 0 \\
-\sin (2 \theta) & 0 & \cos (2 \theta)
\end{array}\right]\left(\begin{array}{l}
\varepsilon \\
\zeta_{\mathrm{h}} \\
\zeta_{\mathrm{v}}
\end{array}\right)_{\mathrm{Lab}}, \\
\Delta \mathbf{k}_{\text {out }}=k\left(\begin{array}{l}
\varepsilon \\
0 \\
0
\end{array}\right)_{\text {Imaging }} .
\end{gathered}
$$

The deviation from the nominal scattering vector becomes

$$
\begin{gathered}
\frac{\Delta \mathbf{Q}}{\left|\mathbf{Q}_{0}\right|}=\frac{\Delta \mathbf{k}_{\mathrm{out}}-\Delta \mathbf{k}_{\mathrm{in}}}{2 k \sin (\theta)} \\
=\frac{1}{2 \sin (\theta)}\left\{\begin{array}{c}
{[1-\cos (2 \theta)] \varepsilon-\sin (2 \theta) \zeta_{\mathrm{v}}} \\
-\zeta_{\mathrm{h}} \\
\sin (2 \theta) \varepsilon-\cos (2 \theta) \zeta_{\mathrm{v}}
\end{array}\right\}_{\text {Imaging }} .
\end{gathered}
$$

If we assume $\zeta_{\mathrm{h}}, \zeta_{\mathrm{v}}$ and $\varepsilon$ to be independent variables, and each to occur randomly with a Gaussian distribution centered about zero, then the corresponding terms should be added in quadrature to estimate the r.m.s. widths in reciprocal space. Let $\Delta \zeta_{\mathrm{v}}, \Delta \zeta_{\mathrm{h}}$ and $\sigma_{\varepsilon}$ be the r.m.s. widths of the corresponding distributions. Then the resulting r.m.s. widths are

$$
\begin{gathered}
\frac{\Delta Q_{\mathrm{rock}^{\prime}}}{\left|\mathbf{Q}_{0}\right|}=\left[\sin ^{2}(\theta) \sigma_{\varepsilon}^{2}+\cos ^{2}(\theta) \Delta \zeta_{\mathrm{v}}^{2}\right]^{1 / 2}, \\
\frac{\Delta Q_{\mathrm{roll}}}{\left|\mathbf{Q}_{0}\right|}=\frac{\Delta \zeta_{\mathrm{h}}}{2 \sin (\theta)}, \\
\frac{\Delta Q_{2 \theta}}{\left|\mathbf{Q}_{0}\right|}=\left\{\cos ^{2}(\theta) \sigma_{\varepsilon}^{2}+\left[\frac{\cos (2 \theta)}{2 \sin (\theta)}\right]^{2} \Delta \zeta_{\mathrm{v}}^{2}\right\}^{1 / 2} .
\end{gathered}
$$

Notably these equations are valid for all points in the sample plane, on-axis as well as off-axis.

In reality the resolution function is far from Gaussian. Similar to a classical triple-axis setup with a monochromator and an analyzer crystal (Rütt et al., 1995), it is characterized by long tails caused by the surface truncation rods - in our case from the two crystals in the monochromator and the sample itself.

\subsection{Reciprocal space mapping described as a convolution}

Having introduced the various terms, we can now give a comprehensive description of the imaging properties of the BFP. We operate in the four-dimensional space spanned by position in sample plane $\mathbf{r}_{\mathrm{s}}$ and reciprocal space coordinates $\mathbf{q}=\left(q_{\text {roll }}, q_{2 \theta}\right)$. The geometrical optics formulation above provides a relation between the measured intensity distribution in the BFP, $I(\mathbf{q})$, the vignetting function in the sample plane, $\operatorname{Vign}\left(\mathbf{r}_{\mathrm{s}}\right)$, the angular attenuation, $\Omega\left(\mathbf{r}_{\mathrm{s}}, \mathbf{q}\right)$, the reciprocal space resolution function, $\operatorname{Res}(\mathbf{q})$, and the field in the sample plane expressed as a distribution function, $R\left(\mathbf{r}_{\mathrm{s}}, \mathbf{q}\right)$. We have

$$
\begin{aligned}
I(\mathbf{q}) \propto & \int_{-\infty}^{\infty} \operatorname{Vign}\left(\mathbf{r}_{\mathrm{s}}\right) \\
& \times \int_{-\infty}^{\infty} R\left(\mathbf{r}_{\mathrm{s}}, \mathbf{q}^{\prime}\right) \Omega\left(\mathbf{r}_{\mathrm{s}}, \mathbf{q}^{\prime}\right) \operatorname{Res}\left(\mathbf{q}-\mathbf{q}^{\prime}\right) \mathrm{d} \mathbf{q}^{\prime} \mathrm{d} \mathbf{r}_{\mathrm{s}}
\end{aligned}
$$

For a suitable small and well centered object, we have Vign $=1$ and $\Omega=\Omega\left(\mathbf{q}^{\prime}\right)$. For some studies the reciprocal resolution function can be considered a delta function. Then equation (22) reduces to

$$
I(\mathbf{q}) \propto \int_{-\infty}^{\infty} R\left(\mathbf{r}_{\mathrm{s}}, \mathbf{q}\right) \exp \left(-\frac{q^{2}}{2 \sigma_{q}^{2}}\right) \mathrm{d} \mathbf{r}_{\mathrm{s}} .
$$

In that case, one image in the BFP simply represents one slice in the reciprocal space map of the entire grain, normalized by $\exp \left(-q^{2} / 2 \sigma_{q}^{2}\right)$. This slice is tilted by $\theta$ with respect to G. Other slices can be added by 'rocking' the sample [cf. equations (8)(10)]. Experimentally, the 'thickness' of such a slice can be increased by integrating the signal over $\theta, e$.g. by a continuous rocking scan.

The range of the reciprocal space map can be enlarged by translating the objective. (A simultaneous translation of the detector in the BFP may be required by the field of view of the detector itself.) It is favorable at the same time to tilt the objective such that the optical axis always points to the same point in the sample plane. For changes of a few degrees in $2 \theta$ or $\eta$ the relevant optical parameters are to a good approximation constant.

\subsection{The effect of coherence}

In order to study the effect of coherence we turn to a Fourier optics description. We shall approximate the incoming beam as a plane wave. The simulations will be based on the use of fractional Fourier transforms, FrFTs (Ozaktas \& Mendlovic, 1995; Le Bolloch et al., 2012). As presented by Pedersen et al. (2018), FrFT calculations can be orders of magnitude faster than traditional Fresnel propagation programs, in particular in connection with the use of a cascade of lenses (a CRL).

Shown in Fig. 2 are the results of an FrFT simulation of a $17 \mathrm{keV}$ study of the pattern in the BFP from an $8 \times 8 \mu \mathrm{m}$ square pinhole placed on the optical axis in the sample plane. The incoming beam is assumed to be parallel with a Gaussian energy spread with $\sigma_{\mathrm{e}}=10^{-4}$, while the geometry of the Be 

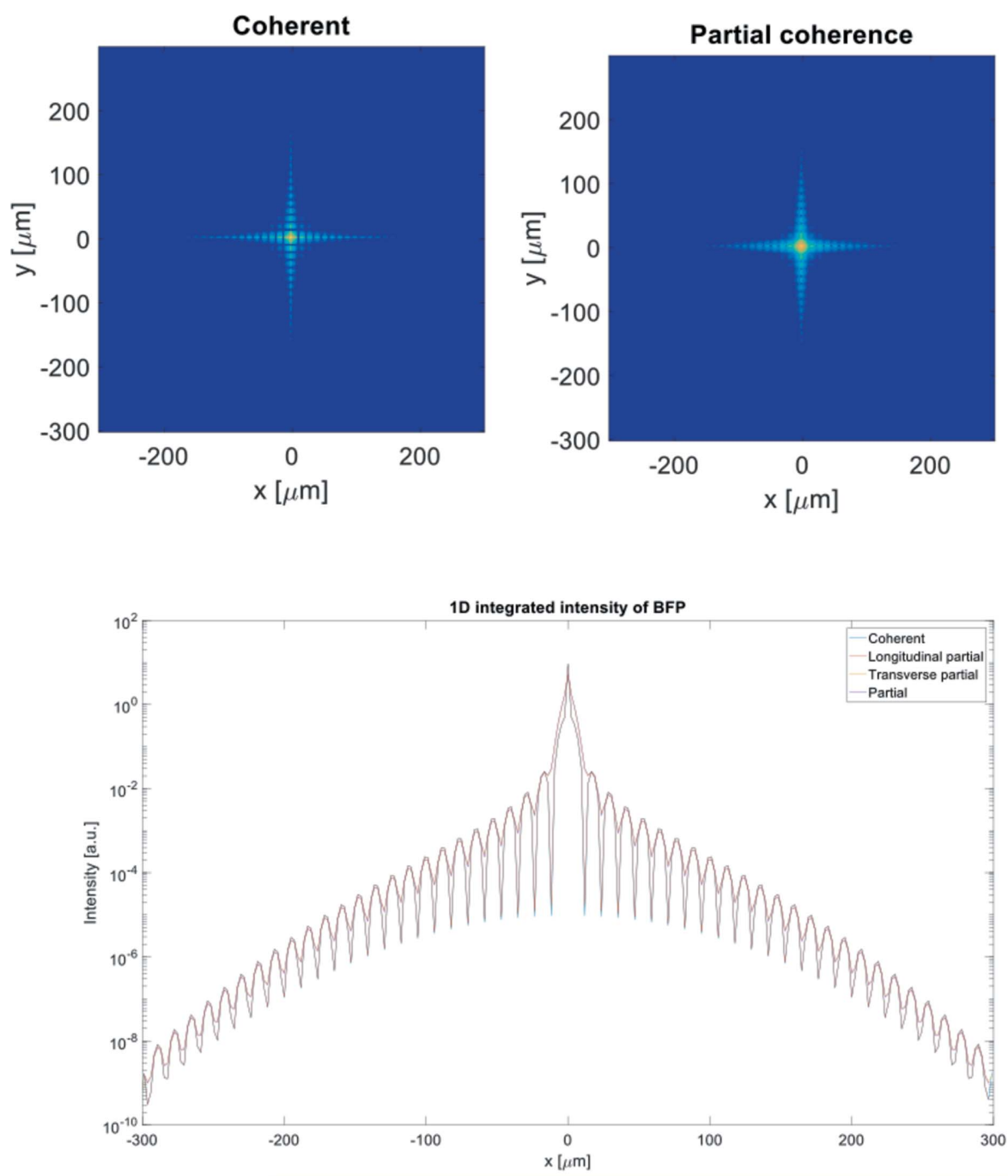

Figure 2

Above: simulated signal in the back focal plane from an $8 \times 8 \mu \mathrm{m}$ square pinhole in the sample plane for a fully coherent and a partially coherent case. Below: a projection onto the horizontal direction for a clearer view of the fringe pattern.

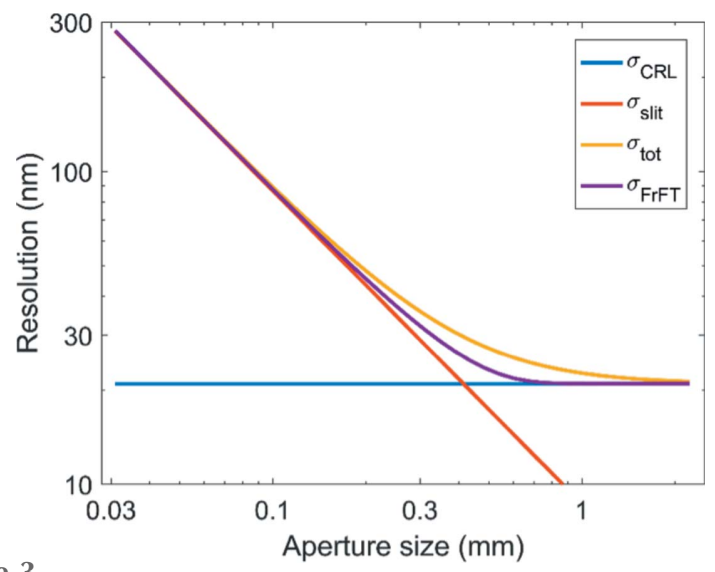

Figure 3

Spatial resolution of an imaging system with a CRL objective and a square aperture placed on the optical axis in the BFP. The figure shows the r.m.s. width in the image plane as a function of the aperture size. The blue, yellow and red lines represent geometrical optics calculations for the CRL alone, for the slit alone and for the combination (see text). Shown in purple is the corresponding wavefield simulation for the entire system based on the use of fractional Fourier transforms.
CRL objective is defined by $N=70, R=$ $50 \mu \mathrm{m}, T=1.6 \mathrm{~mm}, d_{1}=297 \mathrm{~mm}$ and $M=$ 10. Coherence was introduced according to Voelz (2011).

Comparing a fully coherent beam and a beam with a small coherence length (corresponding to placing a $1 \times 1 \mathrm{~mm}$ slit $18 \mathrm{~m}$ upstream from the sample) there is no difference in the image plane, but in the back focal plane a fringe pattern is observed for the coherent case. For partial coherence the fringes disappear and the pattern becomes the envelope of the fully coherent case. Similar to the case for bright-field microscopy (Lyubomirskiy et al., 2016), it appears that one may use back focal plane images for characterizing the degree of vertical and horizontal coherence.

\section{Strain mapping by means of scanning an aperture in the back focal plane}

In this section we shall consider the combination of an aperture in the BFP and imaging with a two-dimensional detector in the image plane. Similarly to operations with transmission electron microscopes, the major advantage of introducing an aperture is that it selects a small region in reciprocal space and that the maps acquired will represent the parts of real space that diffract into this region - without the aperture the region is fixed and given by the numerical aperture (NA) of the objective.

One challenge is that the diffraction limit of the spatial resolution in the imaging plane will deteriorate with decreasing size of the aperture, $D$. Fig. 3 shows results for $17 \mathrm{keV}$ with a parallel incoming beam and with an objective having a focal length $f_{N}=27 \mathrm{~cm}$ and a magnification of 10 . The diffraction limit calculated from geometrical optics is shown for the CRL and the aperture in the BFP independently. The combined effect is not readily determined using geometrical optics, but as a heuristic, the combined effect is modeled as $\sigma_{\text {tot }}=\left(\sigma_{\text {CRL }}^{2}+\sigma_{\text {slit }}^{2}\right)^{1 / 2}$ (yellow curve). Also shown as a purple curve is the result of a corresponding wavefield simulation. In practice, imperfection in lens manufacture currently limits the resolution to around $100 \mathrm{~nm}$. Hence, it appears from Fig. 3 that apertures larger than $80 \mu \mathrm{m}$ will not deteriorate the real space resolution.

We therefore propose to perform strain mapping in the sample plane by scanning a large aperture in the BFP. By a suitable sampling for each voxel in the sample one can derive a reciprocal space map with a strain resolution given by 
equations (19)-(21). Moreover, it is possible to fit the position of a peak to a fraction of the width. As an example, in neutron strain scanning this ratio can be as high as 1:100. Hence a sensitivity to strain variations of $10^{-5}$ or below is clearly within reach.

\section{Experimental demonstration}

The experiments took place at the dedicated dark-field microscopy instrument at ID06, with the goniometer placed $56.66 \mathrm{~m}$ from the source. An $\mathrm{Si}(111)$ Bragg-Bragg monochromator defined a $15.6 \mathrm{keV}$ X-ray beam with an r.m.s. bandwidth of $\sigma_{\mathrm{e}}=6 \times 10^{-5}$. The divergence of the incoming beam was defined by two slits, positioned at distances of 27.8 and $53.9 \mathrm{~m}$ from the source, respectively. The openings of the two slits were both $0.5 \times 0.5 \mathrm{~mm}$, implying divergences (FWHM) of $\Delta \zeta_{\mathrm{v}}=\Delta \zeta_{\mathrm{h}}=0.027 \mathrm{mrad}$.

The experiment was performed in a vertical scattering geometry with $\omega=\eta=0$ ( $c f$. Fig. 1 ). The objective comprised $N=45$ two-dimensional Be lenslets, each with a nominal radius of curvature of $R=50 \mu \mathrm{m}$ and thickness of $T=1.6 \mathrm{~mm}$. Hence, the focal length was $f_{N}=0.406 \mathrm{~m}$. The magnification was determined to be $\mathcal{M}_{\mathrm{CRL}}=12.32$, from which follows $\sigma_{\mathrm{a}}=$ $0.24 \mathrm{mrad}$. The two-dimensional detector placed in the BFP comprised a scintillator coupled by microscope optics to a pco2000 $2 \mathrm{k} \times 2 \mathrm{k}$ CCD with a physical pixel size of $7.4 \mu \mathrm{m}$. The objective and eyepiece in the camera gave an inherent magnification by 10 .

For this setup, 1 pixel $(0.74 \mu \mathrm{m})$ in the back focal plane corresponds to a $\Delta Q / Q$ of $4.0 \times 10^{-6}$. The range in reciprocal space $(\mathrm{FWHM})$ is in the same units $2.35 \sigma_{q}=1.35 \times 10^{-3}$. The FWHM of the vignetting function is $2.35 \sigma_{\mathrm{v}}=5.1 \mathrm{~mm}$.

To test the model for the resolution function of the BFP, an $\mathrm{Si}$ wafer was studied in transmission, with diffraction from a

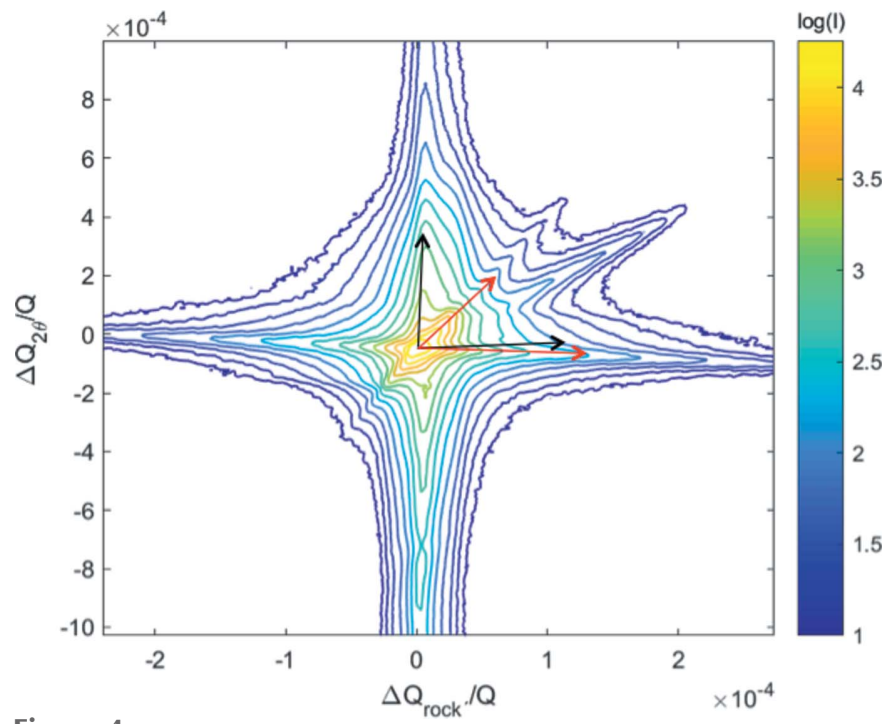

Figure 4

Experimental reciprocal space resolution function. Projected intensity on the scattering plane. For ease of visualization the axes have different ranges. The $\left(q_{\text {rock' }^{\prime}}, q_{2 \theta}\right)$ coordinate system is marked by black arrows and the $\left(q_{\text {rock }}, q_{\|}\right)$coordinate system in red. The contour lines represent a $\log _{10}$ scale as marked by the colorbar to the right.
220 reflection at $2 \theta=23.98^{\circ}$. Scans were made of the 'rocking angle' $\phi$ within a range of $\pm 0.015^{\circ}$ and with 150 equidistant steps. The monochromator was detuned to avoid saturation of the detector.

Three orthogonal projections of the resulting reciprocal space map are shown in Figs. 4, 5 and 6. In the $\left(\hat{q}_{\text {rock }^{\prime}}, \hat{q}_{2 \theta}\right)$ plane there are five streaks, of which two in the up/down direction of the figure are overlapping to some extent. We adopt a similar approach to that used with classical triple-axis diffractometers in a dispersive setup. We can associate one of these streaks - the one along $\hat{q}_{\|}$- with the 'lambda streak' and three of the others with surface streaks from the three Si single crystals in the beam. The one along $\hat{q}_{\text {rock }}$ is from the Si test sample, while the two placed symmetrically around $\hat{q}_{\|}$are assigned to the two monochromator crystals. The strongest streak, however, is along the $\hat{q}_{2 \theta}$ axis. Figs. 5 and 6 reveal that the dominant contribution is an approximately circular disc in the $\left(\hat{q}_{2 \theta}, \hat{q}_{\text {roll }}\right)$ plane in reciprocal space. This is exactly the signature expected of any contribution from the objective. Hence, we attribute this to 'diffuse scattering' from the CRL caused by aberration. Fig. 6 also exhibits additional lowintensity features, in particular in the lower left corner. We tentatively associate these with lens imperfections. Furthermore, we speculate that the lack of inversion symmetry in Fig. 4 is due to the detuning of the monochromator.

Considering only the central part of the resolution function, the experimental values for the FWHM are $\left(\Delta Q_{\text {rock' }^{\prime}} /\left|\mathbf{Q}_{0}\right|\right.$, $\left.\Delta Q_{\text {roll }} /\left|\mathbf{Q}_{0}\right|, \Delta Q_{2 \theta} /\left|\mathbf{Q}_{0}\right|\right)=(13,40,40) 10^{-6}$. The simple Gaussian model provided by equations (19), (20) and 21 gives the corresponding values $(34,44,143) 10^{-6}$. If as a test we insert $\sigma_{\mathrm{e}}=1 \times 10^{-5}$ instead the results are $(18,44,46) 10^{-6}$, which within experimental error is consistent with the experimental data. This finding as well as inspection of Fig. 4 points to a

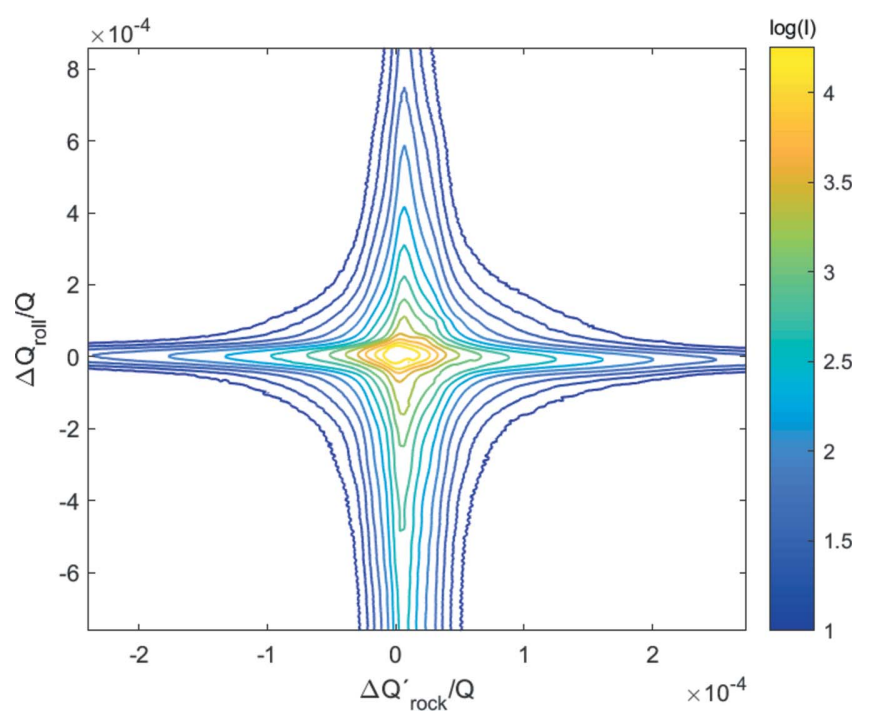

Figure 5

Experimental reciprocal space resolution function. Projected intensity on the $\left(q_{\text {rock' }}, q_{\text {roll }}\right)$ plane. For ease of visualization the axes have different ranges. The contour lines represent a $\log _{10}$ scale as marked by the colorbar to the right. 


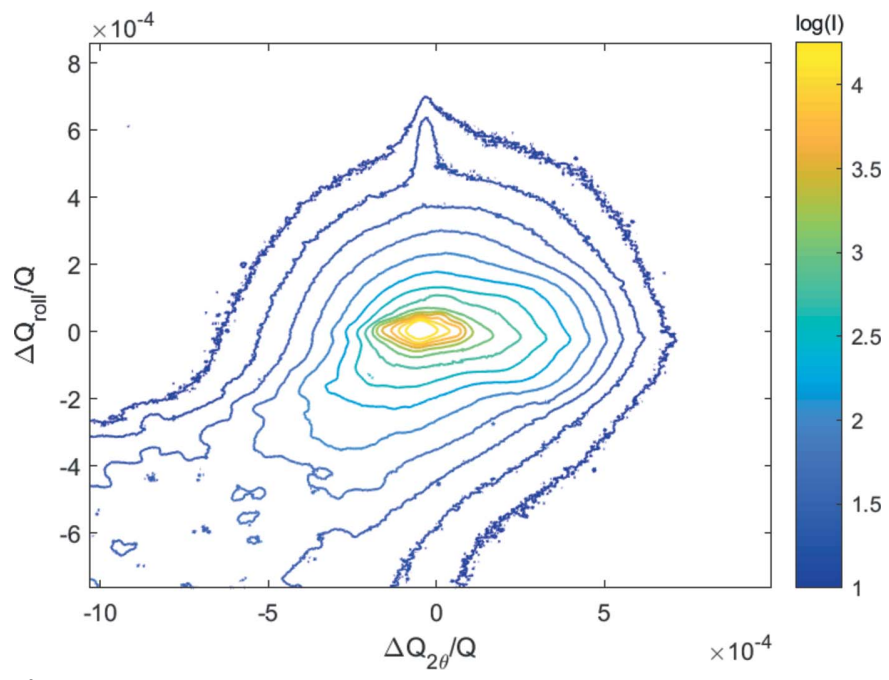

Figure 6

Experimental reciprocal space resolution function. Projected intensity on the $\left(q_{2 \theta}, q_{\text {roll }}\right)$ plane. The axes have identical ranges. The contour lines represent a $\log _{10}$ scale as marked by the colorbar to the right.

much reduced 'lambda streak' in comparison to the simple Gaussian model.

The result is that the FWHM of the reciprocal space resolution function in all directions is small: $40 \times 10^{-6}$ or below.

\section{Discussion}

\subsection{Reciprocal space mapping}

The classical approach to reciprocal space mapping is the use of a triple-axis diffractometer with identical monochromator and analyzer crystals (Pietsch et al., 2004). For a nondispersive setup, where the $d$ spacings of all crystals are nearly the same, the resolution function is as described, for example, by Neumann et al. (1994) and Liss et al. (1998). It is characterized by the presence of three streaks in the scattering plane: a 'sample streak' aligned with $\hat{q}_{\text {rock }}$, a 'monochromator streak' tilted by $\theta$ with respect to $\hat{\boldsymbol{q}}_{\|}$and an 'analyzer streak' tilted by $-\theta$ with respect to $\hat{q}_{\|}$. The dispersive setup is described, for example, by Rütt et al. (1995). Here it is shown that the resolution function tends to be dominated by a streak in the longitudinal direction, the 'lambda streak', but also that all streaks are suppressed.

These features are manifest also in the BFP setting. In particular the 'lambda streak' is suppressed. Further work is required to establish a model that can predict the resolution function in detail. For now we propose to measure it in the manner adopted in this paper, using a semiconductor wafer as a reference sample with a reflection that has a scattering angle close to the one of interest for a given sample.

For reasons of sampling it may be of interest to have a resolution function with identical FWHM along the three principal axes. This can be enabled by varying the incoming divergences and matching the step size in the continuous scan of the rocking angle.
In comparison to the triple-axis configuration the BFP approach has a number of advantages and disadvantages

(1) Three-dimensional mapping. The triple-axis setup is confined to the scattering plane. As such it involves an integration over reciprocal space in the rolling direction. In contrast the resolution function in dark-field microscopy is three dimensional in nature, and may even be designed to be symmetric.

(2) Local information. Dark-field microscopy is favorable for combining reciprocal space mapping with direct space information.

(3) Range. A triple-axis setup can provide a map over a large fraction of reciprocal space. In contrast this is only possible in dark-field microscopy by a complicated combined movement involving both the objective and the BFP detector. Furthermore, owing to the Gaussian-type vignetting term in direct space, it is non-trivial to create a larger reciprocal space map by stitching together smaller parts (unless the sample is an ideal single crystal as is the case for an $\mathrm{Si}$ wafer). A similar challenge relates to mapping in the image plane, as discussed in Paper 1.

An alternative approach to reciprocal space mapping is to avoid using an analyzer and to place the detector in the true far-field (Fraunhofer) regime. This is central to Bragg coherent diffraction imaging type work, but is also used for incoherent beams (see e.g. Jakobsen et al., 2006). The reciprocal space resolution for this case is identical to the BFP case - with the exception of spurious effects by the objective. One subtle difference though is the shift by $\theta$ in which plane is viewed in reciprocal space.

\subsection{Strain scanning}

In Paper 1 it is described that 'mosaicity maps' visualizing the tilt of the diffraction vector (local pole figures) can be generated in two ways: first, by scanning the sample through $(\phi, \chi)$ or linear combinations of these angles, and second, by scanning a combination of the base tilt $\mu$ and a linear combination of $(\phi, \chi)$ that is perpendicular to the beam for all values of $\omega$. With misorientations between neighboring grains or domains typically much larger than the numerical aperture of the objective, this procedure is well adapted to the task.

In Paper 1, a scanning procedure is also introduced for determining the axial strain. This involves scanning the $2 \theta$ arm, that is a combined translation and rotation of the objective and the detector. In this case the $2 \theta$ resolution - as defined by $\sigma_{\mathrm{a}}$ - is of the same order as the strain variation. Hence, one cannot derive a strain distribution for each point in the sample, but only the average strain as determined by the center-of-mass (CMS) of the strain scan.

Operating in the BFP is complementary to this approach as the intrinsic resolution in our case is $5 \times 10^{-5}$. Hence, for many specimens one may determine the entire strain distribution in each voxel. Furthermore, the CMS value of the distribution will be determined with a much higher precision. Also it is easier to ensure a mechanically stable operation by translating an aperture in the BFP than by a combined 
translation of objective and detector. As already mentioned, a disadvantage of the BFP approach is the limitation in strain range. This can to a minor degree be helped by increasing the number of lenslets in the CRL [cf. equation (2)].

\subsection{Limitations and outlook}

It should be emphasized that the approach outlined above only probes reciprocal space in the vicinity of one diffraction vector. Hence, the full orientation of the domains is not determined, and only three out of the nine components of the displacement gradient tensor are monitored (Hofmann et al., 2017). To provide a full description, the mapping has to be repeated for at least two other non-collinear reflections associated with the same domain. This is currently not possible without re-mounting the sample.

Dark-field X-ray microscopy is motivated by the need to generate three-dimensional volumetric data. There are two alternative strategies for obtaining three-dimensional maps. The first is using a one-dimensionally focusing condenser to illuminate a slice of the material, which is then imaged at the oblique angle of $2 \theta$, i.e. a magnified version of classical section topography (Medrano et al., 1997; Ohler et al., 2000). In this case, a three-dimensional volume is obtained in a layer-wise manner by translating the sample through the planar beam in small increments. A second, faster but more involved method involves illuminating the entire grain and taking projections from different viewing angles while rotating the sample about Q (i.e. rotation in $\omega$ ) in the topo-tomography approach (Ludwig et al., 2001). The three-dimensional maps are then reconstructed using adapted tomographic algorithms. The three-dimensional reconstruction algorithm itself, however, is outside the scope of this paper.

In outlook, recently mutilayer Laue lenses (MLLs) have been manufactured with excellent optical performance (Morgan et al., 2015). At ID06 we have successfully tested such devices as objectives for use in dark-field microscopy (to be reported elsewhere). In comparison to CRLs the MLLs have several advantages for operation in the BFP:

(1) Larger numerical aperture. The range in reciprocal space $\sigma_{q}$ increases linearly with $\sigma_{\mathrm{a}}$.

(2) Square aperture. The MLL is optically a thin lens characterized by a square aperture. This implies that for a small sample the detector image is directly proportional to the density in a square in $\left(\hat{q}_{\text {roll }}, \hat{q}_{2 \theta}\right)$ space. This eases interpretation and stitching of partial maps.

(3) Reduced aberration. The dominant diffuse intensity in the $\left(q_{2 \theta}, q_{\text {roll }}\right)$ plane, as shown by Fig. 6 , can be avoided.

The main disadvantage is that two lenses with different focal lengths are needed: one horizontally and one vertically. Hence, their BFPs are not placed at at the same distance. Moreover, the physical aperture of the MLLs manufactured is currently limited to $100 \mu \mathrm{m}$. This implies that the MLL needs to be placed within a few centimetres of the sample to fully exploit the larger NA. This implies a loss in resolution.

We also remark that the concept of a dark-field neutron microscope and its implementation in a time-of-flight opera- tion was proposed by Poulsen et al. (2014). Similar to the X-ray case, dark-field neutron microscopy may be seen as part of a multi-scale approach complementary to neutron absorption tomography and neutron diffraction tomography of grains (Peetermans et al., 2014; Cereser et al., 2017).

\section{Conclusion}

We have derived the relation between reciprocal space and the back focal plane of an X-ray dark-field microscope. Our results enable swapping between mapping in direct space and reciprocal space, which is a key part of many materials studies with transmission electron microscopes. In conjunction with Paper 1, the current article has presented optical tools that enable the transfer of this dual approach to the threedimensional characterization of thick specimens. With analytical expressions for the main optical parameters we hope to ease alignment and to promote dark-field X-ray microscopy as a quantitative tool for materials science.

\section{Acknowledgements}

The authors are grateful to Hugh Simons for stimulating discussions and D. M. Paganin for a critical reading of the manuscript. We thank the ESRF for beamtime.

\section{Funding information}

We thank the instrument center DANSCATT for support of travel. HFP and ACJ acknowledge support by the ERC grant 'Diffraction Based Transmission X-ray Microscopy' (grant No. 291321).

\section{References}

Ahl, S. R., Simons, H., Jakobsen, A. C., Zhang, Y. B., Stöhr, F., Jensen, D. J. \& Poulsen, H. F. (2015). Mater. Sci. Eng. 89, 012016.

Bosak, A., Snigireva, I., Napolskii, K. \& Snigirev, A. (2010). Adv. Mater. 22, 743-751.

Cereser, A. et al. (2017). Sci. Rep. 7, 9561.

Cook, P., Simons, H., Jakobsen, A. C., Yildirim, C., Poulsen, H. F. \& Detlefs, C. (2018). Microsc. Microanal. 24, 88-89.

Ershov, P., Kuznetsov, S., Snigireva, I., Yunkin, V., Goikhman, A. \& Snigirev, A. (2013). J. Appl. Cryst. 46, 1475-1480.

Falch, K. V., Lyubomirsky, M., Casari, D., Snigirev, A., Snigireva, I., Detlefs, C., Michiel, M. D., Lyatun, I. \& Mathiesen, R. H. (2018). Ultramicroscopy, 184, 267-273.

Goodman, J. (2005). Introduction to Fourier Optics. Englewood: Roberts and Company.

Hefferan, C. M., Lind, J., Li, S. F., Lienert, U., Rollett, A. D. \& Suter, R. M. (2012). Acta Mater. 60, 4311-4318.

Hofmann, F., Tarleton, E., Harder, R., Phillips, N., Ma, P.-W., Clark, J. N., Robinson, I., Abbey, B., Liu, W. \& Beck, C. (2017). Sci. Rep. 7, 45993.

Jakobsen, B., Poulsen, H., Lienert, U., Almer, J., Shastri, S., Sørensen, H. O., Gundlach, C. \& Pantleon, W. (2006). Science, 312, 889-892.

King, A., Johnson, G., Engelberg, D., Ludwig, W. \& Marrow, J. (2008). Science, 321, 382-385.

Le Bolloch, D., Pinsolle, E. \& Sadoc, J. F. (2012). Phys. B Phys. Condens. Matter, 407, 3256-3259.

Liss, K.-D., Royer, A., Tschentscher, T., Suortti, P. \& Williams, A. P. (1998). J. Synchrotron Rad. 5, 82-89. 
Ludwig, W., Cloetens, P., Härtwig, J., Baruchel, J., Hamelin, B. \& Bastie, P. (2001). J. Appl. Cryst. 34, 602-607.

Ludwig, W., Reischig, P., King, A., Herbig, M., Lauridsen, E. M., Johnson, G., Marrow, T. J. \& Buffière, J. Y. (2009). Rev. Sci. Instrum. 80, 033905.

Lyubomirskiy, M., Snigireva, I. \& Snigirev, A. (2016). Opt. Express, 24, 13679-13686.

Medrano, C., Rejmánková, P., Ohler, M. \& Matsouli, I. (1997). Nouv. Cim. D, 19, 195-203.

Morgan, A. J., Prasciolu, M., Andrejczuk, A., Krzywinski, J., Meents, A., Pennicard, D., Graafsma, H., Barty, A., Bean, R. J., Barthelmess, M., Oberthuer, D., Yefanov, O., Aquila, A., Chapman, H. N. \& Bajt, S. (2015). Sci. Rep. 5, 9892.

Neumann, H.-B., Rütt, U., Bouchard, R., Schneider, J. R. \& Nagasawa, H. (1994). J. Appl. Cryst. 27, 1030-1038.

Ohler, M., Sanchez del Rio, M., Tuffanelli, A., Gambaccini, M., Taibi, A., Fantini, A. \& Pareschi, G. (2000). J. Appl. Cryst. 33, 1023-1030.

Ozaktas, H. M. \& Mendlovic, D. (1995). J. Opt. Soc. Am. A, 12, 743-751.

Pedersen, A. F., Simons, H., Detlefs, C. \& Poulsen, H. F. (2018). J. Synchrotron Rad. 25, 717-728.

Peetermans, S., King, A., Ludwig, W., Reischig, P. \& Lehmann, E. H. (2014). Analyst, 139, 5767-5771.

Pietsch, U., Holy, V. \& Baumbach, T. (2004). High-Resolution X-ray Scattering. New York: Springer.

Poulsen, H. F. (2004). Three-Dimensional X-ray Diffraction Microscopy. Berlin: Springer.
Poulsen, H. F. (2012). J. Appl. Cryst. 45, 1084-1097.

Poulsen, H. F., Jakobsen, A. C., Simons, H., Ahl, S. R., Cook, P. K. \& Detlefs, C. (2017). J. Appl. Cryst. 50, 1441-1456.

Poulsen, S. O., Poulsen, H. F. \& Bentley, P. M. (2014). Nucl. Instrum. Methods Phys. Res. A, 767, 415-420.

Rütt, U., Neumann, H.-B., Poulsen, H. F. \& Schneider, J. R. (1995). J. Appl. Cryst. 28, 729-737.

Schroer, C., Günzler, T., Benner, B., Kuhlmann, M., Tümmler, J., Lengeler, B. C., Rau, C., Weitkamp, T., Snigirev, A. \& Snigireva, I. (2001). Nucl. Instrum. Methods Phys. Res. A, 467-468, 966-969.

Simons, H., Ahl, S. R., Poulsen, H. F. \& Detlefs, C. (2017). J. Synchrotron Rad. 24, 392-401.

Simons, H., Haugen, A., Jakobsen, A., Schmidt, S., Stohr, F., Majkut, M., Detlefs, C., Daniels, J., Damjanovic, D. \& Poulsen, H. (2018). Nat. Mater. 17, 814-819.

Simons, H., Jakobsen, A. C., Ahl, S. R., Detlefs, C. \& Poulsen, H. F. (2016). MRS Bull. 41, 454-459.

Simons, H., King, A., Ludwig, W., Detlefs, C., Pantleon, W., Schmidt, S., Stöhr, F., Snigireva, I., Snigirev, A. \& Poulsen, H. F. (2015). Nat. Commun. 6, 6098.

Snigirev, A., Kohn, V. G., Snigireva, I. \& Lengeler, B. (1996). Nature, 384, 49-51.

Voelz, D. (2011). Computational Fourier Optics: A MATLAB Tutorial. Bellingham: SPIE.

Williams, D. B. \& Carter, C. B. (2009). Transmission Electron Microscopy. Berlin: Springer. 